\title{
Decompensated congestive heart failure - treatment with levosimendan
}

\author{
Participants: \\ Renata Ferreira Buzzini ${ }^{1}$ \\ (iD) Antonio Silvinato ${ }^{1}$ \\ (D) Idevaldo Floriano ${ }^{1}$ \\ Danderley Marques Bernardo' \\ iD Glauber Romeiro de Almeida
}

Contact: asilvinato@hotmail.com

Final version: January 5th. 2019

1. Brazilian Medical Association, Rua São Carlos do Pinhal, 324 - Bela Vista, São Paulo - SP, Brasil

http://dx.doi.org/10.1590/1806-9282.65.4.524

The Guidelines Project, an initiative of the Brazilian Medical Association, aims to combine information from the medical field in order to standardize producers to assist the reasoning and decision-making of doctors.

The information provided through this project must be assessed and criticized by the physician responsible for the conduct that will be adopted, depending on the conditions and the clinical status of each patient.

\section{INTRODUCTION}

Levosimendan has been extensively evaluated for the treatment of acute heart failure (AHF) and in various other clinical situations characterized by impaired cardiac performance, including heart surgery and sepsis. ${ }^{1}$ Among the drugs generally classified as inotropic, levosimendan was the agent most widely researched over the past 20 years. ${ }^{2}$ It is a drug that belongs to the class of calcium sensitizers and has been commercially available in Brazil since 2002. Through sensitizing action of troponin C to calcium, levosimendan has the potential to improve the cardiac contractility during systole without harming the relaxation during the diastole. It could also have a vasodilating action, which would result in a significant, dose-dependent, improvement of cardiac output (CO) without increasing myocardial oxygen demand. Other hemodynamic effects of the drug on heart failure include increased systolic volume and reduced pulmonary wedge pressure (PWP) and pulmonary arterial pressure. ${ }^{3-5}$ These effects are observed im- mediately after the beginning of the continued intravenous therapy (I.V.) for 24 hs with levosimendan and persist (up to $\approx 10$ days) after the interruption of perfusion, through the action of the long-acting active metabolite OR-1896..$^{3-6}$

\section{OBJECTIVE}

This review aims to assess the impact of the use of levosimendan on the total mortality outcome in patients with dilated cardiomyopathy and ejection fraction lower than $30 \%$ as a method of treatment in comparison with dobutamine.

\section{METHOD}

The method used followed the steps of a systematic review of the literature available to answer the clinical question: What is the impact on the outcome of total mortality of levosimendan in the treatment of 
patients with dilated cardiomyopathy and an ejection fraction $<30 \%$ (heart failure post-intervention or not) when compared to dobutamine?

The question was structured as follows: Patients - Dilated cardiomyopathy and an ejection fraction < 30\% (heart failure post-intervention or not); intervention - I.V. levosimendan; Comparison - dobutamine; Outcomes - Death due to all causes. The search strategies used were: (SIMENDAN OR LEVOSIMENDAN OR DEXTROSIMENDAN) AND DOBUTAMIN* AND (RANDOM* OR SYSTEMATIC[SB]) (MEDLINE / PubMed) and (SIMENDAN OR LEVOSIMENDAN OR DEXTROSIMENDAN) AND DOBUTAMIN (CENTRAL / Cochrane).

All evidence retrieved was evaluated with regards to their risk of biases. For RCTs, we considered: if the question was focal, the randomization appropriate, the allocation blind, double-blind, losses ( $>20 \%)$, prognostic characteristics, outcomes (time, adequacy, measurement), analysis per intention to treat (ITT), sample size calculation, JADAD scale. ${ }^{7}$ After the search for evidence, 120 papers were retrieved, which then had their title and/or summary individually accessed, and of which 28 were selected for evaluation of the full texts. Finally, after evaluating the eligibility criteria, 7 studies were included. The studies included ${ }^{9-15}$ were described in relation to the characteristics of their patients, intervention, comparison and outcome considered (death) to express benefit or harm. The outcome expressed in absolute numbers, absolute risks [absolute risk of the intervention (AR) and absolute risk in comparison (ARC), with differences in risk [reduction (ARR) or increase (ARI) of absolute risk], a confidence interval of 95\% (95\% CI) and number needed to treat (NNT) or to harm (NNH). When there was the presence of the outcome (death) shared by the studies included, the results were expressed through a meta-analysis of data extracted from the selected trials analyzed using the Cochrane software (RevMan 5.3).

\section{RESULTS}

In Table 1, the studies included are described, from which the data were extracted to calculate the meta-analysis (Table 2). The exclusion criteria are available in Figure 1. The studies excluded and the reasons therefor are available in ANNEXES (Table 4).

All evidence retrieved was evaluated according to their risk of biases (Table 3). The individual and global strength of the evidence is expressed in the Synthesis of the Results (Table 5 - ANNEXES)

TABLE 1. CHARACTERISTICS OF THE STUDIES INCLUDED

\begin{tabular}{|c|c|c|c|c|c|}
\hline Study and year & $\begin{array}{l}\text { Patients and number } \\
(\mathrm{N})\end{array}$ & $\begin{array}{l}\text { Intervention } \\
(\mathrm{N})\end{array}$ & $\begin{array}{l}\text { Comparison } \\
(\mathrm{N})\end{array}$ & Outcomes & $\begin{array}{l}\text { Follow-up } \\
\text { time }\end{array}$ \\
\hline $\begin{array}{l}\text { Adamopoulos S, et } \\
\text { al. }{ }^{9} 2006\end{array}$ & $\begin{array}{l}\text { L. ventricular dysfunction } \\
E F<30 \%(46)\end{array}$ & $\begin{array}{l}\text { Levosimendan } \\
6 \mathrm{microg} / \mathrm{kg} \text { attack } \\
0.1 \mathrm{microg} / \mathrm{kg} / \mathrm{min} \text { continuously for } \\
24 \mathrm{~h}(23)\end{array}$ & $\begin{array}{l}\text { Dobutamine } \\
5 \mathrm{microg} / \mathrm{kg} / \mathrm{min} 24 \mathrm{~h} \\
(23)\end{array}$ & Death & 4 months \\
\hline $\begin{array}{l}\text { Alvarez J, et al. }{ }^{\mathbf{1 0}} \\
2006\end{array}$ & $\begin{array}{l}\text { PO of heart surgery with } \\
\text { low cardiac output }(50)\end{array}$ & $\begin{array}{l}\text { Levosimendan } \\
12 \text { microg/kg attack } \\
0.2 \text { microg/kg/min continuously for } \\
24 \mathrm{~h}(25)\end{array}$ & $\begin{array}{l}\text { Dobutamine } \\
7.5 \mathrm{microg} / \mathrm{kg} / \mathrm{min} 24 \mathrm{~h} \\
(25)\end{array}$ & Death & $24 h$ \\
\hline $\begin{array}{l}\text { Bonios MJ, et al. } \\
2012\end{array}$ & $\begin{array}{l}\text { Chronic heart failure EF }< \\
30 \text { resistant to the stan- } \\
\text { dard treatment NYHA } \\
4(42)\end{array}$ & $\begin{array}{l}\text { Levosimendan } \\
0.2 \mathrm{microg} / \mathrm{kg} / \mathrm{min} \\
\text { weekly / } 6 \text { months (21) }\end{array}$ & $\begin{array}{l}\text { Dobutamine } \\
10 \text { microg/kg/min } \\
\text { weekly / } 6 \text { months (21) }\end{array}$ & Death & 6 months \\
\hline $\begin{array}{l}\text { Domingues-Ro- } \\
\text { driguez A, et al. }{ }^{\mathbf{2}} \\
2008\end{array}$ & $\begin{array}{l}\text { Cardiogenic shock (AMI) } \\
\text { after percutaneous inter- } \\
\text { vention. } E F<30(22)\end{array}$ & $\begin{array}{l}\text { Levosimendan - } 24 \text { microg } / \mathrm{kg} \\
\text { attack }-0.1 \mathrm{microg} / \mathrm{kg} / \mathrm{min} \text { contin- } \\
\text { uously for } 24 \mathrm{~h}(11)\end{array}$ & $\begin{array}{l}\text { Dobutamine } \\
5 \mathrm{microg} / \mathrm{kg} / \mathrm{min} 24 \mathrm{~h} \\
(11)\end{array}$ & Death & $24 h$ \\
\hline $\begin{array}{l}\text { Follath F, et al. }{ }^{\mathbf{1 3}} \\
2002\end{array}$ & $\begin{array}{l}\text { Heart failure EF < } 35 \\
(203)\end{array}$ & $\begin{array}{l}\text { Levosimendan - } 24 \text { microg } / \mathrm{kg} \\
\text { attack - } 0.1 \mathrm{microg} / \mathrm{kg} / \mathrm{min} \text { contin- } \\
\text { uously for } 24 \mathrm{~h}(100)\end{array}$ & $\begin{array}{l}\text { Dobutamine } \\
5 \mathrm{microg} / \mathrm{kg} / \mathrm{min} 24 \mathrm{~h} \\
(103)\end{array}$ & Death & $24 \mathrm{~h}, 31 \mathrm{~d}, 180 \mathrm{~d}$ \\
\hline $\begin{array}{l}\text { Levin RL, et al. }{ }^{\mathbf{1 4}} \\
2008\end{array}$ & $\begin{array}{l}\text { PO of revasc. Syndr. Low } \\
\text { Cardiac Output (137) }\end{array}$ & $\begin{array}{l}\text { Levosimendan } \\
10 \text { microg/kg attack } \\
0.1 \mathrm{microg} / \mathrm{kg} / \mathrm{min} \text { continuously for } \\
24 \mathrm{~h}(69)\end{array}$ & $\begin{array}{l}\text { Dobutamine } \\
5 \mathrm{microg} / \mathrm{kg} / \mathrm{min} 24 \mathrm{~h} \\
(68)\end{array}$ & Death & 30 days \\
\hline $\begin{array}{l}\text { Mebazaa A, et al. }{ }^{15} \\
2007\end{array}$ & $\begin{array}{l}\text { Acute heart failure } \\
E F<30\end{array}$ & $\begin{array}{l}\text { Levosimendan } \\
12 \mathrm{microg} / \mathrm{kg} \text { attack } \\
0.1 \mathrm{microg} / \mathrm{kg} / \mathrm{min} \text { continuously for } \\
50 \mathrm{~min} \\
0.2 \mathrm{microg} / \mathrm{kg} / \mathrm{min} 23 \mathrm{~h} \text { (664) }\end{array}$ & $\begin{array}{l}\text { Dobutamine } \\
5 \mathrm{microg} / \mathrm{kg} / \mathrm{min} 24 \mathrm{~h} \\
(663)\end{array}$ & Death & $31 \mathrm{~d} ; 180 \mathrm{~d}$ \\
\hline
\end{tabular}

$\mathrm{NYHA}=$ New York Heart Association functional class; $\mathrm{EF}=$ ejection fraction; $\mathrm{AMI}=$ acute myocardial infarction; $\mathrm{PO}=$ postoperative. 
TABLE 2. RESULTS FOR THE DEATH OUTCOME OF THE SELECTED STUDIES

\begin{tabular}{|c|c|c|c|c|}
\hline \multirow[t]{2}{*}{ Study and year } & \multicolumn{2}{|l|}{ Levosimendan } & \multicolumn{2}{|l|}{ Dobutamine } \\
\hline & Number of deaths & Total of patients & Number of deaths & Total of patients \\
\hline $\begin{array}{l}\text { Adamopoulos S, et } \\
\text { al. }{ }^{\mathbf{9}} 2006\end{array}$ & $2(120 d)$ & 23 & $5(120 d)$ & 23 \\
\hline $\begin{array}{l}\text { Alvarez J, et al. } \\
2006\end{array}$ & $1(24 h)$ & 25 & $1(24 h)$ & 25 \\
\hline $\begin{array}{l}\text { Bonios MJ, et al. }{ }^{\mathbf{1 1}} \\
2012\end{array}$ & 4 (180d) & 21 & $8(180 d)$ & 21 \\
\hline $\begin{array}{l}\text { Domingues-Ro- } \\
\text { driguez A, et al. } \\
2008\end{array}$ & $O(24 h)$ & 11 & $O(24 h)$ & 11 \\
\hline $\begin{array}{l}\text { Follath F, et al. }{ }^{\mathbf{1 3}} \\
2002\end{array}$ & $\begin{array}{l}O(24 h) ; 8(31 d) \\
27(6 \mathrm{~m})\end{array}$ & 103 & $\begin{array}{l}3(24 h) ; 17(31 d) ; \\
38(6 m)\end{array}$ & 100 \\
\hline $\begin{array}{l}\text { Levin RL, et al. }{ }^{\mathbf{1 4}} \\
2008\end{array}$ & $O(24 h) ; 6(30 d)$ & 69 & $\mathrm{O}(24 \mathrm{~h}) ; 17$ (30d) & 68 \\
\hline $\begin{array}{l}\text { Mebazaa A, et al. }{ }^{15} \\
2007\end{array}$ & 79 (31d) $173(6 m)$ & 664 & 91 (31d) 185 (6m) & 663 \\
\hline
\end{tabular}

TABLE 3. DESCRIPTION OF THE BIASES OF THE STUDIES

\begin{tabular}{|c|c|c|c|c|c|c|c|c|c|}
\hline Study and year & Random & $\begin{array}{l}\text { Blinded } \\
\text { alloca- } \\
\text { tion }\end{array}$ & $\begin{array}{l}\text { Dou- } \\
\text { ble-blind }\end{array}$ & Losses & $\begin{array}{l}\text { Prognostic } \\
\text { characteristics }\end{array}$ & Outcomes & $\begin{array}{l}\text { Analysis } \\
\text { by ITT }\end{array}$ & $\begin{array}{l}\text { Sample size } \\
\text { calculation }\end{array}$ & JADAD \\
\hline $\begin{array}{l}\text { Adamopoulos S } \\
2006\end{array}$ & & & & & & & & & 1 \\
\hline Alvarez | 2006 & & & & & & & & & 2 \\
\hline Bonios MJ 2012 & & & & & & & & & 1 \\
\hline $\begin{array}{l}\text { Domingues-Ro- } \\
\text { driguez A } 2008\end{array}$ & & & & & & & & & 1 \\
\hline Follath F 2002 & & & & & & & & & 3 \\
\hline Levin RL 2008 & & & & & & & & & 2 \\
\hline Mebazaa A 2007 & & & & & & & & & 5 \\
\hline
\end{tabular}

$\mathrm{AIT}=$ analysis by intention to treat

absence of biases

no information 


\section{META-ANALYSIS}

Seven RCTs reported the number of deaths in various moments comparing levosimendan versus dobutamine. These studies provided sufficient data for a meta-analysis, considering the results (number of deaths) in these various points or intervals of time of follow-up: 1. Mortality at 24 hours (Figure 2); 2. Mortality at 30 days (Figure 3 ) and 3. Mortality between 120 and 180 days (Figure 4)

Four of the primary trials analyzed death at 24 hours as their outcome. The incidence of death was 0.5\% (1 in 208 patients) in the levosimendan group and $2 \%$ in the dobutamine group (4 in 204 patients). Levosimendan did not reduce the absolute risk of death at 24 hours; there was no statistical significance (ARR1.5\%; 95\% CI -0.6\% to 3.6\%; p = 0.28; $\mathrm{I}^{2}=$ $\mathrm{O} \%, \mathrm{NNT}=\mathrm{NS})$, Figure 2.
Three of the primary trials analyzed death at 30 days as their outcome. Levosimendan reduced the risk of death (ARR) in comparison with dobutamine with values ranging between $2 \%$ and $16 \%$ and an overall reduction of risk equal to $8 \%$; however, there was no statistical significance (95\% CI -0.01 to $0.16 ; p=$ $0.07 ; \mathrm{I}^{2}=70 \%$ ), Figure 3 .

Four of the primary trials analyzed death between 120 and 180 days as their outcome. The incidence of death was $25.4 \%$ (206 in 811 patients) in the levosimendan group and $29.2 \%$ in the dobutamine group (236 in 807 patients). Levosimendan did not reduce the absolute risk of death between 120 and 180 days; there was no statistical significance (ARR $3.8 \%$; $95 \%$ CI - $0.5 \%$ to $8.1 \% ; \mathrm{p}=0.08 ; \mathrm{I}^{2}=28 \%$, NNT $=$ NS), Figure 2 .

FIGURE 2. MORTALITY AT 24 HOURS

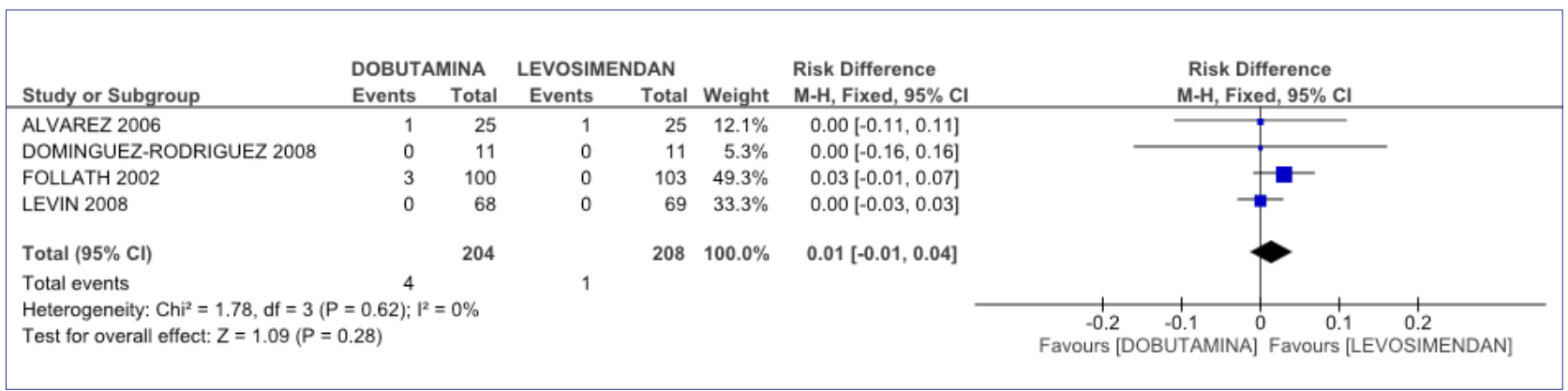

FIGURE 3. MORTALITY AT 30 DAYS

\begin{tabular}{|c|c|c|c|c|c|c|c|}
\hline \multirow[b]{2}{*}{ Study or Subgroup } & \multicolumn{2}{|c|}{ DOBUTAMINA } & \multicolumn{2}{|c|}{ LEVOSIMENDAN } & \multirow[b]{2}{*}{ Weight } & \multirow{2}{*}{$\begin{array}{l}\text { Risk Difference } \\
\text { M-H, Random, } 95 \% \mathrm{Cl}\end{array}$} & \multirow{2}{*}{$\begin{array}{c}\text { Risk Difference } \\
\text { M-H, Random, } 95 \% \mathrm{Cl}\end{array}$} \\
\hline & Events & Total & Events & Total & & & \\
\hline FOLLATH 2002 & 17 & 100 & 8 & 103 & $31.2 \%$ & $0.09[0.00,0.18]$ & $\square$ \\
\hline LEVIN 2008 & 17 & 68 & 6 & 69 & $23.8 \%$ & $0.16[0.04,0.29]$ & $\because$ \\
\hline MEBAZAA 2007 & 91 & 663 & 79 & 664 & $45.0 \%$ & $0.02[-0.02,0.05]$ & \\
\hline Total $(95 \% \mathrm{Cl})$ & & 831 & & 836 & $100.0 \%$ & $0.08[-0.01,0.16]$ & \\
\hline Total events & 125 & & 93 & & & & \\
\hline \multicolumn{7}{|c|}{ Heterogeneity: $\mathrm{Tau}^{2}=0.00 ; \mathrm{Chi}^{2}=6.57, \mathrm{df}=2(\mathrm{P}=0.04) ; \mathrm{I}^{2}=70 \%$} & $\begin{array}{ccccc}-0.2 & -0.1 & 0 & 0.1 & 0.2 \\
\text { Favours [DOBUTAMINA] } & \text { Favours [LEVOSIMENDAN] }\end{array}$ \\
\hline
\end{tabular}

FIGURE 4. MORTALITY BETWEEN 120 AND 180 DAYS

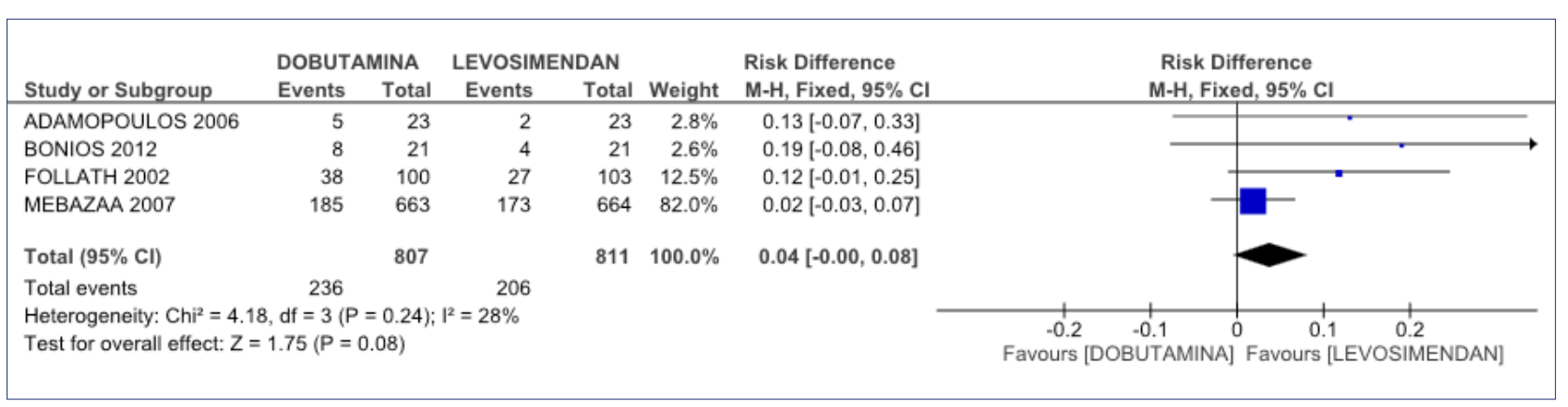




\section{QUALITY OF EVIDENCE (GRADE) PER OUTCOME - TABLE 4 (ANNEXES)}

- Death, 24-hour follow-up (Figure 2) = MODERATE

- Death, 30-day follow-up (Figure 3) = LOW

- Death, 120/180-day follow-up (Figure 4) = LOW

\section{RECOMMENDATION}

In patients with dilated cardiomyopathy and ejection fraction $<30 \%$ (heart failure post-intervention or not), levosimendan does not reduce the risk of death. STRENGTH OF EVIDENCE MODERATE/ LOW

\section{ANNEXES}

TABLE 4. STUDIES EXCLUDED AND REASON

\begin{tabular}{|c|c|}
\hline Study and year & Reason for exclusion \\
\hline 1- Schumann | 2018 & Systematic review \\
\hline 2- Shang G 2017 & Systematic review \\
\hline 3- Kandasamy A 2017 & Does not answer PICO \\
\hline 4- Ishihara S 2016 & Systematic review \\
\hline 5- Kivikko M 2016 & Subgroup analysis of a study included \\
\hline 6- Gong B 2015 & Systematic review \\
\hline 7- Yi GY 2015 & Systematic review \\
\hline 8- Chivite D 2014 & Narrative review \\
\hline 9- Alvarez J 2013 & Intermediary outcome \\
\hline 10- Huang X 2013 & Systematic review \\
\hline 11- Yontar OC 2010 & Intermediary outcome \\
\hline 12- Bergh CH 2010 & Subgroup analysis \\
\hline 13- Duman D 2009 & Intermediary outcome \\
\hline 14- Duygu H 2009 & Intermediary outcome \\
\hline 15- Yilmaz MB 2009 & Intermediary outcome \\
\hline 16- Duygu H 2008 (a) & Intermediary outcome \\
\hline 17- De Hert SG 2008 & Oral formulation \\
\hline 18- Samimi-Fard S 2007 & Does not answer PICO \\
\hline 19- Duygu H 2008 (c) & Intermediary outcome \\
\hline 20- GarcÃa-GonzÃ $j$ lez MJ 2006 & Intermediary outcome \\
\hline 21- Cleland JG 2003 & Economical evaluation \\
\hline
\end{tabular}


TABLE 5. QUALITY OF EVIDENCE (GRADE)

\begin{tabular}{|c|c|c|c|c|c|c|c|c|c|c|c|c|}
\hline \multicolumn{7}{|c|}{ Certainty assessment } & \multicolumn{2}{|c|}{ \# of patients } & \multicolumn{2}{|l|}{ Effect } & \multirow{2}{*}{$\begin{array}{l}\text { Certain- } \\
\text { ty }\end{array}$} & \multirow{2}{*}{$\begin{array}{l}\text { Impor- } \\
\text { tance }\end{array}$} \\
\hline $\begin{array}{l}\text { \# of } \\
\text { studies }\end{array}$ & $\begin{array}{l}\text { Design } \\
\text { of the } \\
\text { study }\end{array}$ & $\begin{array}{l}\text { Risk of } \\
\text { bias }\end{array}$ & $\begin{array}{l}\text { Incon- } \\
\text { sistency }\end{array}$ & $\begin{array}{l}\text { Indirect } \\
\text { evi- } \\
\text { dence }\end{array}$ & $\begin{array}{l}\text { Impre- } \\
\text { cision }\end{array}$ & $\begin{array}{l}\text { Other } \\
\text { consid- } \\
\text { erations }\end{array}$ & $\begin{array}{l}\text { Levosi- } \\
\text { mendan }\end{array}$ & $\begin{array}{l}\text { Dobu- } \\
\text { tamine }\end{array}$ & $\begin{array}{l}\text { Relative } \\
(95 \% \\
\mathrm{CI})\end{array}$ & $\begin{array}{l}\text { Abso- } \\
\text { lute } \\
(95 \% \\
\mathrm{CI})\end{array}$ & & \\
\hline \multicolumn{13}{|c|}{ Mortality at 24 hours (assessed with: Number of deaths in 24 hours) } \\
\hline 4 & $\begin{array}{l}\text { ran- } \\
\text { domized } \\
\text { clinical } \\
\text { trials }\end{array}$ & severe a & $\begin{array}{l}\text { not } \\
\text { severe }\end{array}$ & $\begin{array}{l}\text { not } \\
\text { severe }\end{array}$ & $\begin{array}{l}\text { not } \\
\text { severe b }\end{array}$ & None & $\begin{array}{l}1 / 208 \\
(0.5 \%)\end{array}$ & $\begin{array}{l}4 / 204 \\
(2.0 \%)\end{array}$ & $\begin{array}{l}\text { RR } 3.05 \\
(0.49 \text { to } \\
18.90)\end{array}$ & $\begin{array}{l}40 \text { more } \\
\text { per } \\
1,000 \\
\text { (from } \\
\text { minus } \\
10 \text { to } 351 \\
\text { more) }\end{array}$ & $\begin{array}{l}\oplus \bigoplus \\
\bigoplus \bigcirc \\
\text { MOD- } \\
\text { ERATE }\end{array}$ & $\begin{array}{l}\text { IM- } \\
\text { PORT- } \\
\text { ANT }\end{array}$ \\
\hline \multicolumn{13}{|c|}{ Mortality at 30 days (assessed with: Number of deaths up to 30 days) } \\
\hline 3 & $\begin{array}{l}\text { ran- } \\
\text { domized } \\
\text { clinical } \\
\text { trials }\end{array}$ & $\begin{array}{l}\text { not } \\
\text { severe }\end{array}$ & severe c & $\begin{array}{l}\text { not } \\
\text { severe }\end{array}$ & severe d & None & $\begin{array}{l}93 / 836 \\
(11.1 \%)\end{array}$ & $\begin{array}{l}125 / 831 \\
(15.0 \%)\end{array}$ & $\begin{array}{l}\text { RR } 1.74 \\
\text { (0.94 to } \\
3.19)\end{array}$ & $\begin{array}{l}111 \text { more } \\
\text { per } \\
1,000 \\
\text { (from } \\
\text { minus } 9 \\
\text { to } 329 \\
\text { more) }\end{array}$ & $\begin{array}{l}\oplus \oplus \\
\text { LOW }\end{array}$ & $\begin{array}{l}\text { IM- } \\
\text { PORT- } \\
\text { ANT }\end{array}$ \\
\hline \multicolumn{13}{|c|}{ Mortality between 120 and 180 days (follow-up: variation 120 days to 180 days; assessed with: Number of deaths in this period) } \\
\hline 4 & $\begin{array}{l}\text { ran- } \\
\text { domized } \\
\text { clinical } \\
\text { trials }\end{array}$ & severe e & $\begin{array}{l}\text { not } \\
\text { severe }\end{array}$ & $\begin{array}{l}\text { not } \\
\text { severe }\end{array}$ & severe f & None & $\begin{array}{l}206 / 811 \\
(25.4 \%)\end{array}$ & $\begin{array}{l}236 / 807 \\
(29.2 \%)\end{array}$ & $\begin{array}{l}\text { RR } 1.15 \\
\text { (0.98 to } \\
1.35)\end{array}$ & $\begin{array}{l}44 \text { more } \\
\text { per } \\
1,000 \\
\text { (from } \\
\text { minus } \\
6 \text { to } 102 \\
\text { more) }\end{array}$ & $\begin{array}{l}\oplus \oplus \\
\text { LOW }\end{array}$ & $\begin{array}{l}\text { IM- } \\
\text { PORT- } \\
\text { ANT }\end{array}$ \\
\hline
\end{tabular}

Cl: Confidence interval; RR: Risk ratio - Explanations: a. 50\% with randomization inadequate or not described; only one with blinded allocation, 3 studies without blinding; only with sample size calculation; b. The overall result does not exclude the benefit or harm; c. Test for inconsistency 12 equal to $70 \%$; small overlap of confidence intervals; d. The result does not exclude great harm or benefit; $\mathbf{e}$. 2 studies with JADAD $=1$ and 2 studies with JADAD > = 3; $\mathbf{f}$. The result does not exclude great harm or benefit

\section{REFERENCES}

1. Pollesello P, Parissis J, Kivikko $M$, et al. Levosimendan meta-analyses: is there a pattern in the effect on mortality? Int / Cardiol 2016; 209:77-83.

2. Belletti $A$, Castro ML, Silvetti $S$, et al. The effect of inotropes and vasopressors on mortality: a meta-analysis of randomized clinical trials. $\mathrm{Br}$ ) Anaesth 2015; 115:656-675.

3. García-González M], Jorge-Pérez P, Jiménez-Sosa A, et al. Levosimendan improves hemodynamic status in critically ill patients with severe aortic stenosis and left ventricular dysfunction: an interventional study. Cardiovasc Ther 2015;33:193-199.

4. Innes CA, Wagstaff AJ. Levosimendan: a review of its use in the management of acute decompensated heart failure. Drugs. 2003; 63: 2651-71.

5. Earl GL, Fitzpatrick JT. Levosimendan: a novel inotropic agent for treatment of acute, decompensated heart failure. Ann Pharmacother. 2005; 39: 1888-96.

6. Papp Z, Édes I, Fruhwald S, et al. Levosimendan: molecular mechanisms and clinical implications: consensus of experts on the mechanisms of action of levosimendan. Int J Cardiol 2012;159:82-87.

7. Jadad AR, Moore RA, Carroll D, Jenkinson C, Reynolds DJ, Gavaghan DJ, et al. Assessing the quality of reports of randomized clinical trials: is blinding necessary? Control Clin Trials 1996; 17:1-12

8. Guyatt G, Gutterman D, Baumann MH, Addrizzo-Harris D, Hylek EM, Phillips B et al. Grading strength of recommendations and quality of evidence in clinical guidelines: report from an american college of chest physicians task force. Chest 2006;129(1):174-81. PMID: 16424429

9. Adamopoulos S, Parissis JT, Iliodromitis EK, Paraskevaidis I, Tsiapras D, Farmakis D, et al. Effects of levosimendan versus dobutamine on inflam- matory and apoptotic pathways in acutely decompensated chronic heart failure. Am J Cardiol 2006 1;98:102-6. PMID: 16784930

10. Alvarez J, Bouzada M, FernÃ $j$ ndez AL, Caruezo V, Taboada M, RodrÃguez J, et al. Ginesta [Hemodynamic effects of levosimendan compared with dobutamine in patients with low cardiac output after cardiac surgery]. Rev Esp Cardiol 2006:59:338-45. PMID: 16709386

11. Bonios MJ, Terrovitis JV, Drakos SG, Katsaros F, Pantsios C, Nanas SN, et al. Comparison of three different regimens of intermittent inotrope infusions for end stage heart failure. Int J Cardiol 2012 6;159:225-9. PMID: 21481958

12. Dominguez-Rodriguez A, Samimi-Fard S, Garcia-Gonzalez MJ, Abreu-Gonzalez P. Effects of levosimendan versus dobutamine on left ventricular diastolic function in patients with cardiogenic shock after primary angioplasty. Int J Cardiol 2008 18;128:214-7. PMID: 17643535

13. Follath F, Cleland JG, Just H, Papp JG, Scholz H, Peuhkurinen K, et al. Efficacy and safety of intravenous levosimendan compared with dobutamine in severe low-output heart failure (the LIDO study): a randomised double-blind trial. Lancet 2002 20;360:196-202. PMID: 12133653

14. Levin RL, Degrange MA, Porcile R, Salvagio F, Blanco N, Botbol AL, et al. [The calcium sensitizer levosimendan gives superior results to dobutamine in postoperative low cardiac output syndrome]. Rev Esp Cardiol 2008;61:471-9. PMID: 18462650

15. Mebazaa A, Nieminen MS, Filippatos GS, Cleland JG, Salon JE, Thakkar $R$, et al. Levosimendan vs. dobutamine: outcomes for acute heart failure patients on beta-blockers in SURVIVE. Eur J Heart Fail 2009 ;11:304-11. PMID: 19158152 